\title{
Ultrasound Elastography in Thyroid Lesions
}

\author{
Neeraj Prajapati ${ }^{1}$, S.K. Rastogi ${ }^{2}$ \\ ${ }^{1}$ Associate Professor, Department of Radiodiagnosis, ${ }^{2}$ Associate Professor, Department of Radiodiagnosis, SRMS IMS Bareilly, \\ UP, India
}

Corresponding author: Dr S.K. Rastogi, F-132, SRMS IMS campus, Bhojipura, Bareilly, India

DOI: http://dx.doi.org/10.21276/ijcmsr.2019.4.1.20

How to cite this article: Neeraj Prajapati, S.K. Rastogi. Ultrasound elastography in thyroid lesions. International Journal of Contemporary Medicine Surgery and Radiology. 2019;4(1):A81-A84.

\section{A B S T R A C T}

Introduction: Ultrasound elastography is a novel technique which non-invasively detects the hardness of a mass. It is being applied in evaluation of various thyroid, breast and other masses to know the probability of malignancy within them on the basis of tissue stiffness. Study aimed to know the effectiveness of ultrasound elastography in differentiating benign and malignant thyroid nodules with a good diagnostic accuracy.

Material and methods: This was a prospective study including fifty patients. All the patients underwent high resolution ultrasound of the thyroid nodules along with elastography examination. The nodules were given score from one to five on the basis of their stiffness on elastography. All patients with thyroid nodules underwent FNAC as well.

Results: On real time ultrasound elastography Score 1 was assigned to even strain or elasticity in the entire lesion which was seen in 20 cases. Score of 2 was assigned to strain or elasticity in most of the lesion with some areas of no strain which was seen in 16 cases. Score of 3was assigned to strain or elasticity at the peripheral part of the lesion sparing the central part of the lesion which was seen in 05 cases. Score of 4 was assigned to no strain or elasticity in the entire lesion which was seen in 07 cases. Score of 5 was assigned to no strain or elasticity in the entire lesion or in the surrounding area which was seen in 2 cases. Out of 50 cases on real time ultrasound elastography 41 patients presented with a benign nodule and 09 presented with a malignant nodule. On FNAC 42 patients were diagnosed as benign where as 08 patients were diagnosed as malignant. Conclusion: Real time ultrasound elastography can be used to differentiate between benign and malignant thyroid nodules with a good diagnostic accuracy. Score of 1 to 3 suggests benign lesions where as a score of 4 or 5 suggests malignant lesions on real time ultrasound elastography.

Keywords: Ultrasound Elastography, Thyroid Nodule, FNAC (Fine Needle Aspiration Cytology)

\section{INTRODUCTION}

Thyroid nodule is the result of abnormal growth of thyroid cells containing benign or malignant cells within the thyroid gland. The main clinical concern for managing thyroid nodules is to exclude malignant diseases which account for $4 \%$ to $6 \%$ of all thyroid benign nodules. ${ }^{1}$

The last decade has seen two important advances i.e. high resolution ultrasonography and Fine Needle Aspiration Cytology (FNAC). ${ }^{2}$ The detection rate of smaller thyroid nodules can be increased using high-resolution ultrasonography. As compared with FNAC, high resolution ultrasonography has the advantage of being a non invasive procedure and giving immediate information. ${ }^{3}$

Conventional ultrasound does not provide direct information corresponding to the hardness of a nodule. So ultrasound elastography was developed to obtain information on tissue stiffness noninvasively. This technique can evaluate the degree of distortion of a tissue under application of an external force and is based on the principle that the softer parts of tissues deform easier than the harder parts under compression, thus allowing an objective determination of tissue stiffness. The thyroid gland is well positioned for elastographic examination. It can be easily assessed and efficiently compressed against normal underlying anatomic structures with an ultrasound probe. ${ }^{4}$

To enhance the diagnostic ability of sonography, ultrasound elastography, including static and real-time elastography has been introduced into the clinic with an aim of improving the diagnosis of thyroid nodules..$^{5}$ A thyroid nodule that is clinically firm to hard and in consistency is associated with an increased risk of malignancy. The principle of elastography imaging is based on evaluating differential tissue characteristics regarding rigidity versus elasticity. Elastography is used complementary to conventional ultrasonography for improving the diagnosis of thyroid tumors that appear harder than the surrounding tissue. ${ }^{6}$

Real-time ultrasound elastography is one of the most widely used techniques. It is a newly developed dynamic technique that reflects the deformation or distortion of tissue in response to the application of an external compression load. Elastography combines the detection advantages of high resolution ultrasound with cancer diagnostic information. On the elastogram, different colour modes imply different tissue stiffness, thereby offering more information with respect to differentiation between benign and malignant lesions. ${ }^{7}$ Study aimed to know the effectiveness of ultrasound 
elastography in differentiating benign and malignant thyroid nodules with a good diagnostic accuracy.

\section{MATERIAL AND METHODS}

The present study was a prospective study of fifty patients conducted between 2016 and 2018. An informed written consent was obtained for participation in the study. Demographic characteristics, clinical presentation, and ultrasound findings of each patient were noted. On the basis of presenting symptoms and radiologic investigations, surgical intervention was done with removal of the lesion. Specimens obtained were subjected to detailed histopathological analysis. Patients' records were carefully reviewed, and a correlation between histopathological findings and outcome following the surgery was done.

The ultrasound was performed on a higher end ultrasound machine having capability of color doppler and sonoelastography and a linear array transducer with a centre frequency of 7.5 MHz (range, 5.0-14.0 MHz).The patient was positioned on his or her back with the neck slightly extended over a pillow. Conventional B-mode ultrasound images of the thyroid nodules are initially obtained. The diagnostic parameters are size, shape, borders, internal echo, calcification, rear-echo change and the halo ring of the tumour. Colour Doppler ultrasound was carried out in all patients to evaluate the vascularity of the thyroid lesion.

The dual mode is used to display real-time elastogram and the grey-scale ultrasound images simultaneously. Color scale is used to evaluate the elastography images: red indicated 'hard tissue', green indicated 'medium stiffness' of the tissue and blue indicated 'soft tissue'. The US elastographic image was matched with an elasticity color scale and classified using the Rago elasticity score. ${ }^{3}$ Score 1 is assigned to even strain or elasticity in the entire lesion. Score of 2 is assigned to strain or elasticity in most of the lesion with some areas of no strain, Score of 3 is assigned to strain or elasticity at the peripheral part of the lesion sparing the central part of the lesion, Score of 4 is assigned to no strain or elasticity in the entire lesion, Score of 5 is assigned to no strain or elasticity in the entire lesion or in the surrounding area.

After obtaining detailed clinical and radiological data, the patients were subjected to FNAC (direct or under USG guidance).

\section{RESULTS}

This study included total of fifty patients out of which twenty six were female and twenty four were male with age range between eighteen to eighty years. Majority of the patients presented with mass in the region of thyroid gland which moves with deglutition. All the patients underwent thyroid elastography followed by FNAC of the thyroid nodule. The elastography findings were co related with the FNAC findings.

On real time ultrasound elastography Score 1 (Fig. 1) was assigned to even strain or elasticity in the entire lesion which was seen in 20 cases. Score of 2(Fig. 2) was assigned to strain or elasticity in most of the lesion with some areas of no strain which was seen in 16 cases. Score of 3 was assigned to strain or elasticity at the peripheral part of the lesion sparing the

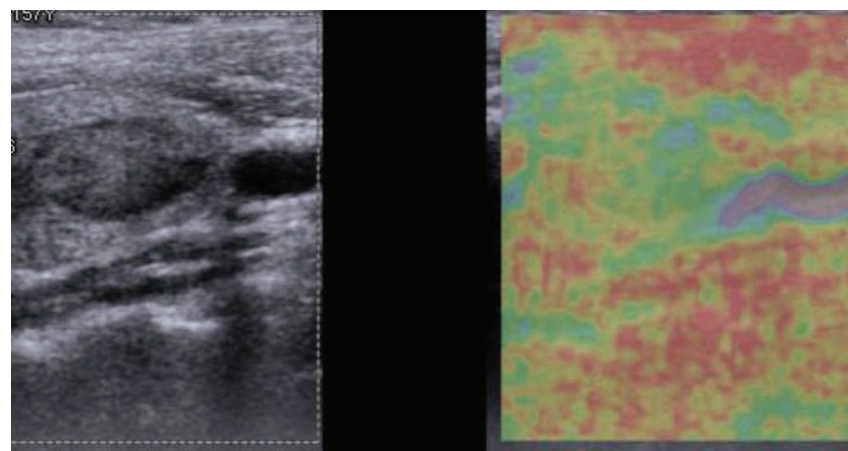

Figure-1: Even elasticity in the entire thyroid nodule. (Score 1)

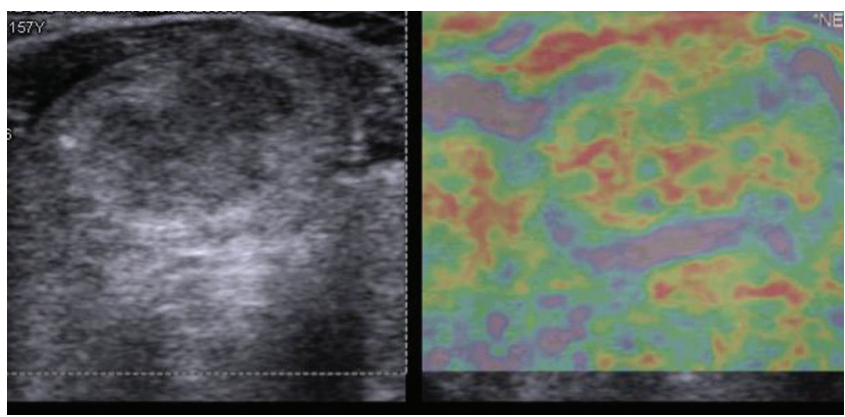

Figure-2: Elasticity in most of the lesion with some areas of no strain. (Score 2 )

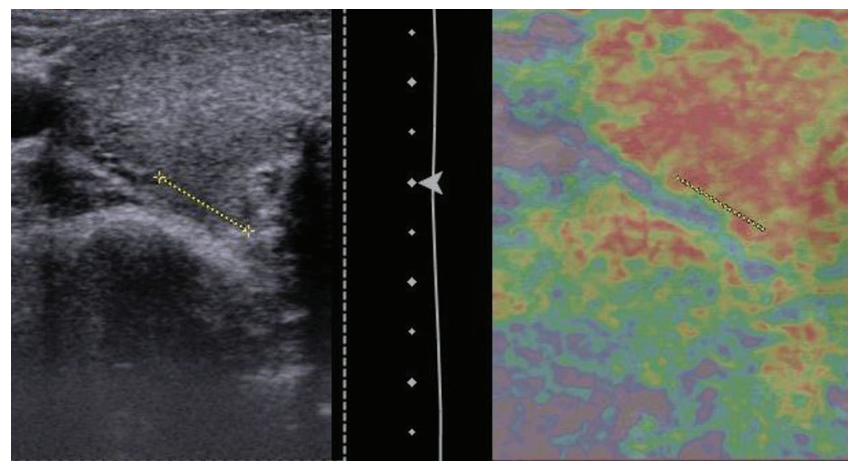

Figure-3: No strain or elasticity in the entire lesion. (Score 4)

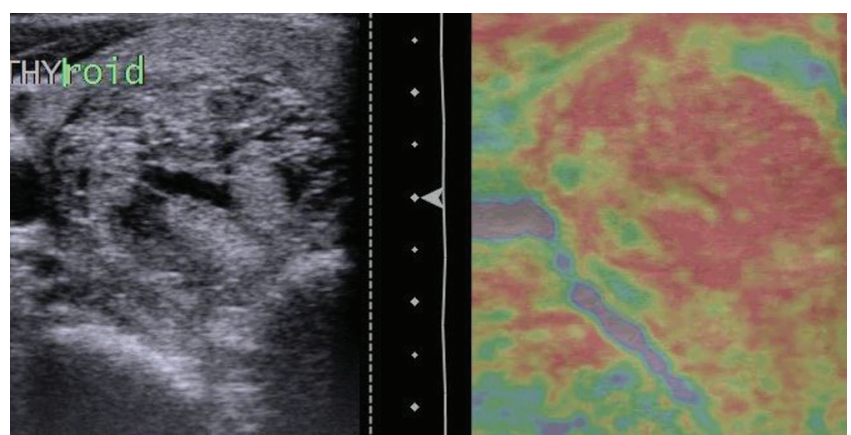

Figure-4: No strain or elasticity in the entire lesion and in the surrounding area. (Score 5)

central part of the lesion which was seen in 05 cases. Score of 4 (Fig. 3) was assigned to no strain or elasticity in the entire lesion which was seen in 07 cases. Score of 5(Fig. 4) was assigned to no strain or elasticity in the entire lesion or in the surrounding area which was seen in 2 cases.

All of the 50 patients underwent FNAC (direct or ultrasound 
guided) under aseptic precautions. 42 patients were diagnosed as benign where as 08 patients were diagnosed as malignant. Out of 50 cases on real time ultrasound elastography 41 patients presented with a benign nodule and 09 presented with a malignant nodule.

Out of 42 patients diagnosed as having benign nodules, 19 patients had colloid goitre with associated cystic degeneration, 18 had colloid goitre, 3 had benign adenomatous nodule, 1 had autoimmune thyroiditis and 1 patient had lymphocytic thyroiditis.

Out of 8 patients diagnosed as having malignant nodules, 4 had papillary carcinoma, 3 had follicular carcinoma and 1 patient had medullary carcinoma.

On correlation of FNAC findings with real time ultrasound elastography findings, Out of the 50 cases, there was 1 false positive diagnosis of malignancy, ie nodule of 1 patient was diagnosed as malignant on elastography but was found out to be benign on FNAC.

Real time ultrasound elastography score of 1 to 3 were classified as benign and score of 4 to $5(\mathrm{P}<0.001)$ were classified as malignant. The correlation coefficient is calculated to be 0.8 .

Thus, the elasticity scores $4-5$ were highly predictive of malignancy with a sensitivity of $97.6 \%$, a specificity of $66.6 \%$, a positive predictive value of $88.8 \%$, and a negative predictive value of $97.6 \%$.

\section{DISCUSSION}

Elastography is a technique that uses ultrasound to analyze the stiffness of a nodule by measuring the amount of distortion that occurs when the nodule is subjected to external pressure. The elasticity scoring system initially proposed by Ueno and Itoh was useful for comparing breast ultrasound elastographic results. ${ }^{8}$

Hong $\mathrm{Y}$ et al examined one hundred forty-five nodules in 90 patients by B-mode ultrasound, color doppler ultrasound, and ultrasound elastography. The final diagnosis was obtained from histologic findings. Tissue stiffness was scored from 1 to 6 on the basis of subjective analysis of the elastograms. On real-time ultrasound elastography, 86 of 96 benign nodules (90\%) had a score of 1 to 3 , whereas 43 of 49 malignant nodules (88\%) had a score of 4 to $6(\mathrm{P}<.001)$, with sensitivity of $88 \%$, specificity of $90 \%$, a positive predictive value of $81 \%$, and a negative predictive value of $93 \%$. High sensitivity (88\%) and specificity (93\%) were also observed in 68 nodules that had a greatest diameter of $1 \mathrm{~cm}$ or less. Hence concluding real-time ultrasound elastography is a promising imaging technique that is useful in the differential diagnosis of thyroid cancer. ${ }^{9}$

Elastography examines the mechanical and elastic properties of soft tissue, which rely on the composition and structural organization of the macromolecules. Some pathologic conditions induce considerable changes in the soft tissue structure, modifying the elastic properties and leading to increased firmness and reduced mobility of the involved tissue. Malignant thyroid nodules tend to be much harder than benign ones, such as papillary thyroid carcinoma, the most common histologic type of thyroid malignancy. Papillary thyroid carcinoma has complex papillae with a central fibrovascular stalk. Psammoma bodies and fibrosis are often found in them. ${ }^{10,11}$

There was 1 false positive diagnosis of malignancy in our series, ie nodule of 1 patient was diagnosed as malignant on elastography but was found out to be benign on FNAC. This patient was given a score of 4 on elastography. On co relative FNAC examination that nodule turned out to be benign adenomatous nodule with hemorrhagic degeneration. The nodule behaved as hard on elastography may be because of hemorrhagic degeneration.

Recently a meta-analysis was performed in 2014 by Sun J et al to assess the diagnostic power of ultrasound elastography in differentiating benign and malignant thyroid nodules for elasticity score and strain ratio assessment. A total of 5481 nodules in 4468 patients for elasticity score studies and 1063 nodules in 983 patients for strain ratio studies were analyzed. The overall mean sensitivity and specificity of ultrasound elastography for differentiation of thyroid nodules were 0.79 (95\% confidence interval [CI], 0.77-0.81) and 0.77 (95\% CI, 0.76-0.79) for elasticity score assessment and 0.85 (95\% CI, 0.81-0.89) and 0.80 (95\% CI, 0.770.83 ) for strain ratio assessment, respectively. These results confirmed those obtained in the previous meta-analysis. Ultrasound elastography has high sensitivity and specificity for identification of thyroid nodules. It is a promising tool for reducing unnecessary fine-needle-aspiration biopsy. ${ }^{12}$

The drawback of our study was its small sample size and co relation was done with FNAC.

\section{CONCLUSION}

Real time ultrasound elastography can be used to differentiate between benign and malignant thyroid nodules with a good diagnostic accuracy. Score of 1 to 3 suggests benign lesions where as a score of 4 or 5 suggests malignant lesions on real time ultrasound elastography.

If used along with the gray scale and color Doppler sonography, it can potentially reduce unnecessary interventions for the diagnosis of lesions. Further it can reduce morbidity associated with these procedures

As the sample size was small in our study, prospective studies with larger sample size and histopathological co relation are required. Further being a new diagnostic technique a multicentre trial of elastography should be performed for its authentication.

\section{REFERENCES}

1. SunJ,CaiJ,WangX. Real-time Ultrasound Elastography for differentiation of Benign and Malignant Thyroid Nodules. J Ultrasound Med 2014; 33(1):495-502

2. Ross DS. Evaluation of thyroid nodule. J Nucl Med 1991;32(3):2181 -92

3. Rago T, Santini F et al.Elastography: New Developments in Ultrasound for Predicting Malignancy in Thyroid Nodules. The Journal of Clinical Endocrinology \& Metabolism 2007;92(6): 2917-2922.

4. Refaat R, Kamel A, Elganzory M, Awad NM. Can real time ultrasound elastography using color score and strain ratio differentiate between benign and malignant solitary thyroid nodules. The Egyptian Journal of Radiology and Nuclear Medicine 2014;45(2): 75-87. 
5. Zhang Y, He Y, Xu HX, Lui C, Guo LH, Lui LN et al: Virtual Touch Tissue Imaging on Acoustic Radiation Force Impulse Elastography. J Ultrasound Med 2014; 33(3):585-95

6. Stoian D, Cornianu M: Nodular thyroid cancer. Diagnostic value of real time elastography. Chirurgia 2012;107: 39-46

7. Wang Y, Dan H-J, Dan H-Y, Li T, Hu. Differential Diagnosis of Small Single Solid Thyroid Nodules using real time ultrasound elastography. Journal of International Medical Research 2010; 38(1):466

8. Ueno E, Itoh A. Diagnosis of breast cancer by elasticity imaging (EI). Eizo Joho Med 2004; 36(4):1-6.

9. Hong Y, Liu X, Li Z, Zhang X, Chen M, Luo Z: Real time ultrasound elastography in the differential diagnosis of benign and malignant thyroid nodule. J.Ultrasound med 2009; 28(6): 861-867.

10. Carcangui ML, Zampi G, Rosai J. Papillary thyroid carcinoma: a study of its many morphologic expressions and clinical correlates. Pathol Annu 1985; 20(2):1-44.

11. Reading CC, Charboneau JW, Hay ID, Sebo TJ. Sonography of thyroid nodules: a "classic pattern" diagnostic approach. Ultrasound Q 2005; 21(3):157165.

12. SunJ,CaiJ,WangX. Real-timeUltrasound Elastography for Differentiation of Benign and Malignant Thyroid Nodules: A Meta-analysis. J Ultrasound Med. 2014;33(3):495-502.

Source of Support: Nil; Conflict of Interest: None

Submitted: 25-01-2019; Accepted: 02-03-2019; Published online: 15-03-2019 